\title{
Optical coherence tomography retinal nerve fiber layer analysis in eyes with long axial lengths
}

This article was published in the following Dove Press journal:

Clinical Ophthalmology

\author{
Sagar B Patel ${ }^{1,2}$ \\ Nisha Reddy ${ }^{2}$ \\ Xihui Lin ${ }^{1,2}$ \\ Jess T Whitson ${ }^{1,2}$ \\ 'Department of Ophthalmology, \\ University of Texas Southwestern \\ Medical Center, Dallas, TX, USA; \\ ${ }^{2}$ Department of Ophthalmology, \\ University of Texas Southwestern \\ Medical School, Dallas, TX, USA
}

Correspondence: Jess T Whitson Department of Ophthalmology, University of Texas Southwestern Medical School, 5323 Harry Hines Blvd, Dallas, TX 75390, USA

Tel + I 2I 46483826

Email jess.whitson@utsouthwestern.edu
Purpose: To evaluate the relationship between axial length (AL) and retinal nerve fiber layer (RNFL) profile and to characterize differences in optical coherence tomography RNFL of myopic glaucomatous eyes compared to nonglaucomatous eyes.

Methods: Retrospective chart review of 170 eyes of 89 subjects with optical biometry and optical coherence tomography RNFL assessment was conducted.

Results: Temporal RNFL thickness showed no association with AL in either glaucomatous or nonglaucomatous eyes. Nasal thinning was most strongly associated with glaucoma in myopic eyes. Both myopic glaucomatous and nonglaucomatous eyes had a mean RNFL thickness of 16-22 $\mu \mathrm{m}$ thinner than mean RNFL thickness of normal AL eyes.

Conclusion: An average of 16-22 $\mu \mathrm{m}$ thinning of RNFL compared to nomogram can be tolerated in patients with long AL. Prominent nasal thinning likely represents changes from axial elongation. Temporal RNFL thinning in those with long AL tends to be mild, and significant thinning should raise suspicion for glaucoma.

Keywords: myopia, axial length, ocular coherence tomography retinal nerve fiber layer (OCT RNFL), glaucoma

\section{Introduction}

Patients with long axial lengths (ALs) are at increased risk for glaucoma. ${ }^{1}$ The clinical diagnosis of glaucoma in patients with long ALs is difficult due to inherent changes to optic disc morphology as the eye elongates.

Assessment of the peripapillary retinal nerve fiber layer (RNFL) thickness is an important component in detecting structural damage in patients with glaucoma. Imaging devices such as optical coherence tomography (OCT) allow for the quantitative and objective assessment of the peripapillary RNFL thickness. ${ }^{2}$ Multiple studies have shown that peripapillary RNFL thickness measurements using OCT can detect glaucoma with manifest visual-field defects with a high sensitivity and specificity. ${ }^{3,4}$ However, the interpretation of OCT RNFL in long AL eyes is difficult due to frequent abnormal findings encountered.

Conflicting data exist regarding the influence of myopia on peripapillary RNFL thickness. The study by Hoh et $\mathrm{al}^{5}$ has shown no associations, whereas others have found that more long AL eyes had thinner RNFL measured by OCT. ${ }^{6,7}$

Another issue is the topographic profile of the RNFL in subjects with long AL eyes. When evaluated with OCT, such eyes are often classified outside normal limits in the nasal sectors. ${ }^{8}$ Long AL eyes might have a topographic profile of the RNFL that differs from that of normal AL eyes with more pronounced thinning in the nasal sectors. This may be important in the use of OCT for detecting glaucomatous damage, 
because the current OCT technology determines the damage by comparing the measured RNFL thickness with a nonmyopic normative database.

The purpose of this study was to evaluate the relationship between the AL and peripapillary RNFL thickness profile as well as to characterize the difference in OCT RNFL between glaucomatous and nonglaucomatous eyes with long ALs.

\section{Methods}

The study protocol was approved by the Institutional Review Board of the University of Texas Southwestern School of Medicine before the study began. The study was a retrospective case review of all patients seen at Parkland Memorial Hospital between the dates of August 1, 2010 and April 1, 2016. Medical records of patients with optical biometry were identified (IOL Master, Carl Zeiss Meditec and Alcon Lenstar; Haag Streit AG, Koeniz, Switzerland) to obtain AL data. Of these, those that had an OCT RNFL done were identified. All subjects had complete ophthalmic exams including measurement of intraocular pressure by applanation, cycloplegic refraction, assessment of the cup-to-disc ratio, and automated visual-field examinations (Humphrey visual field analyzer with SITA standard 24-2; Carl Zeiss Meditec, Jena, Germany). All eyes underwent OCT examination of the peripapillary RNFL using Spectralis Tracking Laser Tomography (Heidelberg Engineering HRA OCT, Heidelberg, Germany). As subjects were deidentified, and HIPAA compliance was ensured for each step. Subjects were stratified into glaucoma vs nonglaucoma groups: glaucomatous eyes were defined as those with a glaucomatous visual-field defect and enlarged cup-to-disc ratio or asymmetric ratios regardless of intraocular pressure. Additionally, all patients were stratified into normal and long AL groups; normal AL group having an $\mathrm{AL}<25 \mathrm{~mm}$ and the long $\mathrm{AL}$ group having an $\mathrm{AL} \geq 25 \mathrm{~mm}$.
Subjects or eyes with a history of any optic nerve disorder or significant posterior segment pathology such as ischemic optic neuropathy, nonischemic optic neuropathy, multiple sclerosis, infiltrative nerve processes, and tractional retinal detachments were excluded.

Linear regression was used to determine the correlation between RNFL thickness and continuous variables (AL, spherical equivalent, and age). A Student's $t$-test was used to determine significant associations between RNFL thickness in the glaucoma vs nonglaucoma group and between the long and short AL group. Significance was defined as $p<0.05$.

\section{Results \\ Participants}

A total of 170 eyes were examined from a total of 89 subjects in the study. Sixty-one were female and 28 were male, with a racial breakdown of: 4.39\% Asian, 47.26\% Black, $4.39 \%$ Caucasian, and $43.96 \%$ Hispanic. The mean age was 68.10 years (range 30-90 years) with an average AL of 23.85 (range $21.88-29.86 \mathrm{~mm}$ ) and average refractive error of -1.00 diopter (range -10.5 to 3.5 diopters). The subjects were categorized into 2 groups: a long AL group ( $n=18$ eyes, average AL $26.46 \mathrm{~mm}$, range $25-29.62 \mathrm{~mm}$ ) and a normal AL group ( $\mathrm{n}=152$ eyes, average AL $23.56 \mathrm{~mm}$, range 21.88-24.98 mm). Each group was subsequently broken up into nonglaucoma and glaucoma groups. Table 1 summarizes the demographic characteristics.

\section{OCT measurements}

The mean, superior, nasal, and inferior quadrants were significantly different between the normal and glaucomatous eyes in both the long AL and normal AL groups (all $p<0.05$, Table 2). The temporal quadrant showed a significant difference between the normal and glaucomatous eyes within the normal AL group, but a difference was not found between

Table I Characteristics of the subjects

\begin{tabular}{|c|c|c|c|c|c|c|c|}
\hline & \multicolumn{3}{|c|}{ Long AL group ( $\mathrm{N}=18$ eyes) } & \multicolumn{4}{|c|}{ Normal AL group ( $\mathrm{N}=\mid \mathrm{I} 2$ eyes) } \\
\hline & Normal (N=I I) & Glaucoma $(\mathbf{N}=7)$ & $p$-value ${ }^{a}$ & Normal $(\mathrm{N}=86)$ & Glaucoma $(\mathbf{N}=66)$ & $p$-value ${ }^{b}$ & $p$-value \\
\hline Age (years) & $58.64 \pm 5.39$ & $68.00 \pm 5.66$ & $<\mathbf{0 . 0 0 1}$ & $66.08 \pm 9.73$ & $53.49 \pm 8.40$ & $<0.001$ & $<\mathbf{0 . 0 0 1}$ \\
\hline Females, no (\%) & $3(27.27)$ & $4(57.14)$ & 0.18 & $60(65.93)$ & $5 I(77.27)$ & 0.35 & 0.01 \\
\hline IOP (mmHg) & $14.40 \pm 1.65$ & $19.14 \pm 4.74$ & 0.01 & $15.49 \pm 3.53$ & $|5.9| \pm 6.94$ & 0.19 & 0.60 \\
\hline Refraction (diopters) & $-5.29 \pm 3.67$ & $-2.93 \pm 2.87$ & 0.24 & $-0.48 \pm 2.36$ & $-0.73 \pm 2.24$ & 0.55 & $<\mathbf{0 . 0 0 I}$ \\
\hline $\mathrm{AL}(\mathrm{mm})$ & $26.84 \pm 1.96$ & $26.84 \pm 0.75$ & 0.23 & $23.63 \pm 0.7 \mid$ & $23.47 \pm 0.81$ & 0.19 & $<\mathbf{0 . 0 0 I}$ \\
\hline Cup-to-disc ratio & $0.63 \pm 0.17$ & $0.79 \pm 0.21$ & 0.09 & $0.55 \pm 0.15$ & $0.70 \pm 0.19$ & $<0.001$ & 0.10 \\
\hline Mean deviation $(\mathrm{db})$ & $-11.06 \pm 9.90$ & $-22.18 \pm 9.57$ & 0.06 & $-6.75 \pm 6.93$ & $-10.97 \pm 7.63$ & 0.001 & 0.004 \\
\hline PSD (dB) & $3.58 \pm 3.25$ & $5.29 \pm 2.51$ & 0.32 & $4.430 \pm 2.89$ & $5.33 \pm 3.60$ & 0.08 & 0.42 \\
\hline
\end{tabular}

Notes: The data are given as the mean \pm standard deviation. ${ }^{a}$ Value for comparing nonglaucoma and glaucoma in the long AL group. ${ }^{b} \mathrm{Value}$ for comparing nonglaucoma and glaucoma in the normal AL group. 'Value for comparing myopia and nonmyopia. Statistically significant values are shown in bold.

Abbreviations: AL, axial length; IOP, intraocular pressure; PSD, pattern standard deviation. 
Table 2 RNFL thickness in normal and glaucoma eyes in long AL and normal AL groups

\begin{tabular}{|c|c|c|c|c|c|c|c|}
\hline \multirow[b]{2}{*}{ RNFL thickness $(\mu \mathrm{m})$} & \multicolumn{3}{|c|}{ Long AL group $(\mathrm{N}=18)$} & \multicolumn{4}{|c|}{ Normal AL group $(N=\mid 52)$} \\
\hline & Normal (N=II) & Glaucoma (N=7) & $p$-value ${ }^{a}$ & Normal $(\mathbf{N}=86)$ & Glaucoma $(\mathbf{N}=66)$ & $p$-value ${ }^{b}$ & $p$-value ${ }^{c}$ \\
\hline Mean & $74.82 \pm 12.77$ & $50.29 \pm 15.64$ & $<0.001$ & $90.37 \pm 10.92$ & $72.53 \pm 15.66$ & $<0.001$ & $<0.001$ \\
\hline Superior & $85.73 \pm 11.29$ & $62.14 \pm 15.55$ & $<0.001$ & $111 \pm 18.93$ & $88.50 \pm 23.49$ & $<0.001$ & $<0.001$ \\
\hline Nasal & $54.00 \pm 16.1$ & $27.43 \pm 14.90$ & $<0.001$ & $69.73 \pm 11.89$ & $57.44 \pm 16.82$ & $<0.001$ & $<0.001$ \\
\hline Inferior & $88.09 \pm 18.54$ & $59.86 \pm 24.31$ & 0.01 & $113.92 \pm 18.86$ & $87.42 \pm 24.47$ & $<0.001$ & $<0.00 \mathrm{I}$ \\
\hline Temporal & $71.18 \pm 30.09$ & $51.14 \pm 17.84$ & 0.13 & $67.21 \pm 14.86$ & $58.73 \pm 16.76$ & $<0.001$ & 0.98 \\
\hline Superior/inferior & $0.99 \pm 0.13$ & $1.12 \pm 0.38$ & 0.33 & $0.99 \pm 0.18$ & $1.04 \pm 0.32$ & 0.23 & 0.60 \\
\hline Nasal/temporal & $0.82 \pm 0.31$ & $0.60 \pm 0.46$ & 0.23 & $1.09 \pm 0.30$ & $1.03 \pm 0.40$ & 0.30 & $<0.00 \mathbf{I}^{\mathrm{d}}$ \\
\hline
\end{tabular}

Notes: a Value for comparing normal and glaucoma in the long AL group. bValue for comparing normal and glaucoma in the normal AL group. ${ }^{\mathrm{c} V a l u e}$ for comparing normal and long $A L$ eyes without glaucoma. ${ }^{d}$ Ratio long $A L: 0.73 \pm 0.37 ; N / T$ ratio normal $A L: 1.06 \pm 0.34$. Statistically significant values are shown in bold.

Abbreviations: $A L$, axial length; RNFL, retinal nerve fiber layer.

normal and glaucomatous eyes within the long AL group. When comparing the long AL group to the normal AL group as a whole, a significant difference was found in all ocular tomography regions but the temporal quadrant (Table 2).

Table 3 shows the correlation among demographic variables like biometric and refractive error and mean OCT RNFL thickness within nonglaucomatous eyes and glaucomatous eyes. In the nonglaucomatous eyes, age, sex, spherical equivalent, and cup-to-disc ratio were all associated with mean RNFL thickness, with the strongest association being spherical equivalent. Glaucomatous eyes demonstrated strong correlation between the cup-to-disc ratio and mean deviation to RNFL thickness. Refractive error was not associated with mean RNFL thickness in glaucomatous eyes. No significant associations were found between RNFL thickness and gender in either group.

When comparing AL to RNFL thickness, a significant negative association was found between AL and RNFL thickness in most quadrants in the nonglaucomatous eyes, with the strongest association in the inferonasal and inferior

Table 3 Pearson correlation coefficients of the relationship between RNFL layer thickness and subject characteristics in nonglaucoma vs glaucoma eyes

\begin{tabular}{|c|c|c|}
\hline & \multicolumn{2}{|c|}{ Mean RNFL $(\mu \mathrm{m})$} \\
\hline & $\begin{array}{l}\text { Nonglaucoma } \\
\text { group }(n=97)\end{array}$ & $\begin{array}{l}\text { Glaucoma } \\
\text { group }(n=73)\end{array}$ \\
\hline Age (years) & $0.255(0.012)$ & $-0.038(0.75)$ \\
\hline Sex & $(0.340)$ & $(0.091)$ \\
\hline Refraction (diopters) & $0.383(<0.001)$ & $0.122(0.303)$ \\
\hline Cup-to-disc ratio & $-0.237(0.019)$ & $-0.380(<0.001)$ \\
\hline IOP (mmHg) & $-0.038(0.7 \mathrm{II})$ & $0.101(0.394)$ \\
\hline Mean deviation $(\mathrm{db})$ & $0.23(0.023)$ & $0.570(<0.001)$ \\
\hline PSD (db) & $0.007(0.945)$ & $-0.355(0.003)$ \\
\hline
\end{tabular}

Notes: The data are given as the Pearson correlation coefficients with $p$-value in parenthesis. Significant values are bold, $p$-value $<0.05$.

Abbreviations: IOP, intraocular pressure; PSD, pattern standard deviation; RNFL, retinal nerve fiber layer. quadrants (Table 4). Temporal RNFL thickness showed no significant associations with AL in either group.

\section{Discussion}

Previous studies on the determinants of RNFL thickness in the normative database of the ocular coherence tomography noted a significant decrease in RNFL thickness with increasing AL, equal to $2.2 \mu \mathrm{m} / \mathrm{mm} .{ }^{6}$ The normative database was not significantly different from ours with a mean $\mathrm{AL}$ of $23.8 \mathrm{~mm}$ and refractive error of -0.54 diopters. But the normative database for the Heidelberg Spectralis OCT excluded highly myopic patients with spherical equivalents of $>-7$ diopters and included 201 healthy white subjects. ${ }^{9}$ Due to the relatively restricted normative database, many OCT RNFL results flagged as abnormal do not necessarily represent real disease. ${ }^{10}$ One such group frequently flagged is with long ALs. With increasing AL, the superotemporal

Table 4 Pearson correlation coefficients of the relationship between axial length and RNFL thickness in nonglaucoma vs glaucoma eyes

\begin{tabular}{|c|c|c|}
\hline \multirow[b]{2}{*}{ RNFL thickness $(\mu \mathrm{m})$} & \multicolumn{2}{|l|}{ Axial length (mm) } \\
\hline & $\begin{array}{l}\text { Nonglaucoma } \\
\text { group }(n=97)\end{array}$ & $\begin{array}{l}\text { Glaucoma } \\
\text { group }(n=73)\end{array}$ \\
\hline Mean & $-0.408(<0.001)$ & $-0.245(0.037)$ \\
\hline Superior & $-0.428(<0.001)$ & $-0.220(0.06 I)$ \\
\hline Nasal & $-0.364(0.001)$ & $-0.350(0.002)$ \\
\hline Inferior & $-0.449(<0.001)$ & $-0.180(0.128)$ \\
\hline Temporal & $0.168(0.099)$ & $-0.016(0.893)$ \\
\hline ST & $-0.299(0.002)$ & $-0.029(0.808)$ \\
\hline SN & $-0.407(<0.001)$ & $-0.379(<0.001)$ \\
\hline IN & $-0.505(<0.001)$ & $-0.256(0.029)$ \\
\hline IT & $-0.24 \mid(0.017)$ & $-0.083(0.485)$ \\
\hline
\end{tabular}

Notes: The data are given as the Pearson correlation coefficients with $p$-value in parenthesis. Significant values are bold, $p$-value $<0.05$.

Abbreviations: IN, inferonasal; IT, inferotemporal; RNFL, retinal nerve fiber layer; $\mathrm{SN}$, superonasal; ST, superonasal. 


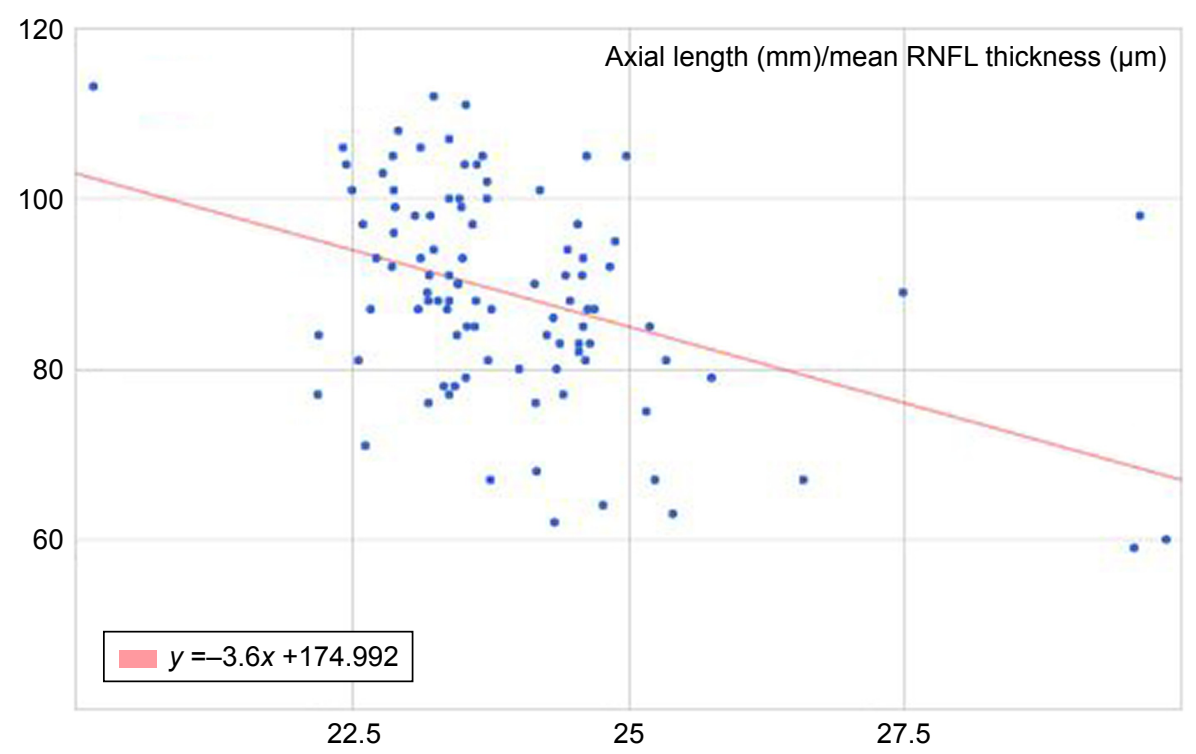

Figure I Linear regression analysis of the relationship of mean RNFL thickness with AL.

Notes: $Y$ axis represents $A L$ in $\mathrm{mm}$. $X$ axis represents mean RNFL thickness in micrometers. $R^{2}=0.17, y=-3.6 x+174$.

Abbreviations: $A L$, axial length; RNFL, retinal nerve fiber layer.

and inferotemporal RNFL bundles tend to converge temporally (Figure 1). ${ }^{11}$ This can result in a temporal shift of the superior and inferior RNFL bundle peaks while maintaining normal magnitude.

Consistent with previous studies, we found an inverse relationship between increasing ALs and average RNFL thickness. We found a decrease in mean RNFL thickness of $3.6 \mu \mathrm{m} / \mathrm{mm}$, which is slightly higher than that previously published of $2 \mu \mathrm{m} / \mathrm{mm}$ and $2.75 \mu \mathrm{m} / \mathrm{mm}$ (Figure 2) ${ }^{6,14}$ The key area of thinning occurs nasally when ALs increase. Relatively, the temporal RNFL is thicker as AL increases, as demonstrated by the nasal RNFL thickness/temporal RNFL thickness (N/T) ratio of less-than-one in long AL eyes. This is consistent with the theory of a temporal shift of the axial bundles in the myopic eyes. The temporal RNFL is not less affected by axial elongation, but rather thickness in eyes with high myopia due to the temporal shift of axial bundles. Also consistent with the theory is the significant thinning in the superior and inferior nerve bundles.

As expected, we found that when comparing glaucomatous eyes to nonglaucomatous eyes in normal AL eyes, the glaucomatous eyes have a significantly thinner RNFL and higher mean deviation on HVF. In both glaucomatous and nonglaucomatous groups, the long $\mathrm{AL}$ groups had a mean RNFL thickness of about 16-22 $\mu \mathrm{m}$ thinner compared to the normal AL groups. Clinically, such a degree of decrease from the normative data on the machines may be attributable to changes from elongation rather than true glaucoma. Also of note is the $18-25 \mu \mathrm{m}$ mean difference in eyes with and without glaucoma in both normal and long AL groups.

Some previous studies did not find significant associations between AL and RNFL thickness. ${ }^{5,12,13}$ These studies were done using earlier generation time-domain OCT and confocal laser devices which may have limited their resolution and lowered sensitivity. While overall, the correlation between ALs is not strong (Pearson coefficient $\left[R^{2}\right]$ of 0.17 ), there is a trend toward thinner RNFL as AL increase, and when AL of $25 \mathrm{~mm}$ is used to divide the groups the difference is very significant. A value of $25 \mathrm{~mm}$ was chosen because it is 2 standard deviations from the mean on most normative data. ${ }^{6}$ The difference in these 2 methods of analysis can be explained by a potential threshold phenomenon in that temporal migration of nerve bundles only occurs after a certain AL.

\section{Limitations}

There were several limitations to this study. Although well powered for our analysis, the long AL group is relatively small. A large group may provide additional insights. Another limitation of the study is its inability to show long-term changes in the patient. A longitudinal study, as opposed to a cross-sectional study, could better show the anatomic changes over time in the patients studied. Our definition of glaucoma by HVF does not include pre-perimetric glaucoma patients. However, this does not change our analysis significantly since we were mainly interested in the difference between the groups. Additionally, a fixed effect of both eye-included subjects may have caused a bias in the data. 


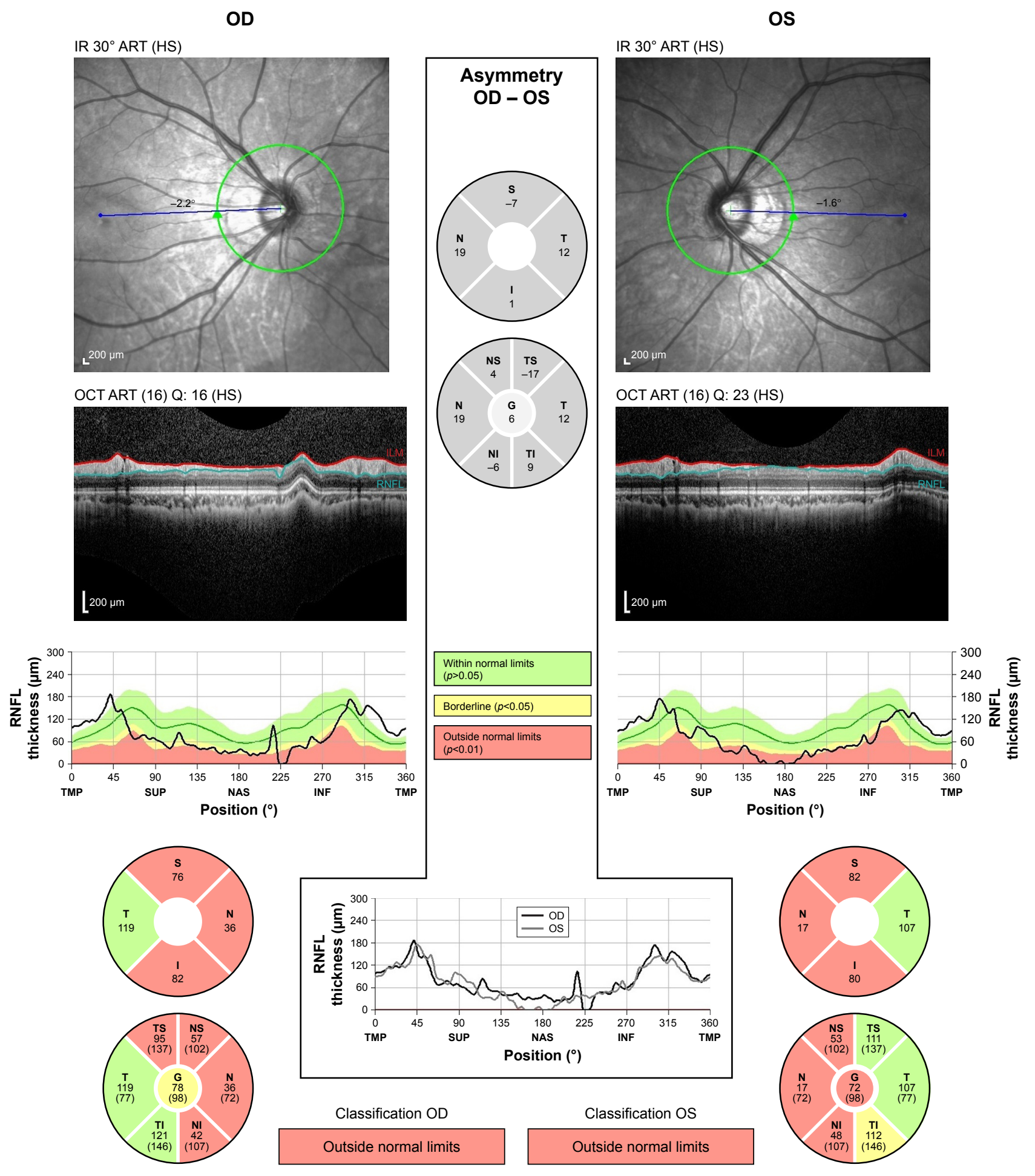

Figure 2 Typical RNFL of nonglaucomatous long $A L$ subject $(A L=28 \mathrm{~mm})$.

Note: Note the significant nasal thinning with normal temporal thickness.

Abbreviations: AL, axial length; RNFL, retinal nerve fiber layer; TMP, temporal; SUP, superior; NAS, nasal; INF, inferior.

\section{Conclusion}

In evaluating RNFL of patients with long AL, a 16-22 $\mu \mathrm{m}$ average thinning compared to the nomogram can be tolerated and prominent nasal thinning likely represent changes from axial elongation. Temporal RNFL thinning in those with long AL tends to be mild, and any significant thinning in this area should raise suspicion for glaucomatous changes. 


\section{Acknowledgments}

This work was supported in part by an unrestricted grant from Research to Prevent Blindness (New York, NY, USA). The abstract of this paper was presented at the November 12, 2017 American Academy of Ophthalmology Conference as a poster presentation with interim findings. This work was approached as a retrospective chart review. Patient consent to review their medical records was not required by the institutional review board due to the retrospective nature of the study. Patient confidentiality was ensured by assigning patients a unique randomized identification number, and by removing all identifiable patient data. The database was stored on an encrypted, password-protected drive and only available to those conducting the study.

\section{Disclosure}

The authors report no conflicts of interest in this work.

\section{References}

1. Mitchell P, Hourihan F, Sandbach J, Wang JJ. The relationship between glaucoma and myopia: the Blue Mountains Eye Study. Ophthalmology. 1999;106(10):2010-2015.

2. Aref AA, Budenz DL. Spectral domain optical coherence tomography in the diagnosis and management of glaucoma. Ophthalmic Surg Lasers Imaging. 2010;41:S15-S27.

3. Grewal DS, Tanna AP. Diagnosis of glaucoma and detection of glaucoma progression using spectral domain optical coherence tomography. Curr Opin Ophthalmol. 2013;24(2):150-161.

4. Chang RT, Knight OJ, Feuer WJ, Budenz DL. Sensitivity and specificity of time-domain vs spectral-domain optical coherence tomography in diagnosing early to moderate glaucoma. Ophthalmology. 2009;116(12):2294-2299.
5. Hoh ST, Lim MC, Seah SK, et al. Peripapillary retinal nerve fiber layer thickness variations with myopia. Ophthalmology. 2006;113(5): 773-777.

6. Budenz DL, Anderson DR, Varma R, et al. Determinants of normal retinal nerve fiber layer thickness measured by Stratus OCT. Ophthalmology. 2007;114(6):1046-1052.

7. Choi SW, Lee SJ. Thickness changes in the fovea and peripapillary retinal nerve fiber layer depend on the degree of myopia. Korean $J$ Ophthalmol. 2006;20(4):215-219.

8. Vernon SA, Rotchford AP, Negi A, Ryatt S, Tattersal C. Peripapillary retinal nerve fibre layer thickness in highly myopic Caucasians as measured by Stratus optical coherence tomography. Br J Ophthalmol. 2008;92(8):1076-1080.

9. Silverman AS, Hammel N, Khachatryan N, et al. Diagnostic accuracy of the spectralis and cirrus reference databases in differentiating between healthy and early glaucoma eyes. Ophthalmology. 2016; 123(2):408-414.

10. Leite MT, Rao HL, Weinreb RN, et al. Agreement among spectraldomain optical coherence tomography instruments for assessing retinal nerve fiber layer thickness. Am J Ophthalmlol. 2011;151(1):85-92.

11. Leung CK, Yu M, Weinreb RN, et al. Retinal nerve fiber layer imaging with spectral-domain optical coherence tomography: interpreting the RNFL maps in healthy myopic eyes. Invest Ophthalmol Vis Sci. 2012;53(11): 7194-7200.

12. Leung CK, Mohamed S, Leung KS, et al. Retinal nerve fiber layer measurements in myopia: an optical coherence tomography study. Invest Ophthalmol Vis Sci. 2006;47(12):5171-5176.

13. Garcia-Valenzuela E, Anderson NG, Pons M, Iezzi R. Retinal thickness by OCT in subjects with emmetropia, hyperopia, and myopia. Invest Ophthalmol Vis Sci. 2002;43:2574.

14. Kashiwagi K, Tamura M, Abe K, Kogure S, Tsukahara S. The influence of age, gender, refractive error, and optic disc size on the optic disc configuration in Japanese normal eyes. Acta Ophthalmol Scand. 2000;78:200-203.
Clinical Ophthalmology

\section{Publish your work in this journal}

Clinical Ophthalmology is an international, peer-reviewed journal covering all subspecialties within ophthalmology. Key topics include: Optometry; Visual science; Pharmacology and drug therapy in eye diseases; Basic Sciences; Primary and Secondary eye care; Patient Safety and Quality of Care Improvements. This journal is indexed on Submit your manuscript here: http://www.dovepress.com/clinical-ophthalmology-journal

\section{Dovepress}

PubMed Central and CAS, and is the official journal of The Society of Clinical Ophthalmology (SCO). The manuscript management system is completely online and includes a very quick and fair peer-review system, which is all easy to use. Visit http://www.dovepress.com/ testimonials.php to read real quotes from published authors. 